\title{
OPTIMAL BUNCHING WITHOUT OPTIMAL CONTROL
}

\author{
Georg Nöldeke \\ Department of Economics \\ University of Bonn \\ Adenauerallee 24-42 \\ 53113 Bonn, Germany \\ georg.noldeke@uni-bonn.de
}

\author{
Larry Samuelson
}

Department of Economics

University of Wisconsin

1180 Observatory Drive

Madison, WI 53706-1393 USA

LarrySam@ssc.wisc.edu

\section{January 17, 2006}

Running head: Optimal Bunching

Address correspondence to:

Larry Samuelson

Department of Economics

University of Wisconsin

1180 Observatory Drive

Madison, WI 53706-1393

LarrySam@ssc.wisc.edu

608-263-7791 (voice), 608-262-2033 (fax)

\footnotetext{
${ }^{1}$ This is a revised version of "Decomposable Principal-Agent Problems." We thank two referees and an associate editor for helpful comments. Financial support from the Deutsche Forschungsgemeinschaft, GRK 629 and SFB TR 15 at the University of Bonn, and the National Science Foundation, SES-0241506, is gratefully acknowledged.
} 


\title{
OPTIMAL BUNCHING WITHOUT OPTIMAL CONTROL
}

\begin{abstract}
This paper presents simple sufficient conditions under which optimal bunches in adverse-selection principal-agent problems can be characterized without using optimal control theory.
\end{abstract}

Keywords: Principal-Agent Problem, Bunching, Nonlinear Pricing, Assignment Approach

Journal of Economic Literature numbers C60, C70, D40, D80. 


\title{
OPTIMAL BUNCHING WITHOUT OPTIMAL CONTROL
}

\author{
by Georg Nöldeke and Larry Samuelson
}

\section{Introduction}

This paper considers adverse selection principal-agent models with quasi-linear utility functions and a one-dimensional decision variable (in addition to monetary transfers). The type of the agent is one dimensional and continuously distributed. The agent's preferences satisfy a singlecrossing property.

When the monotonicity constraint on incentive-compatible decision functions implied by the single crossing property binds, the optimal decision function features a bunch, i.e., there is an interval of types choosing the same decision. In such cases, the standard analysis relies on control theory to characterize optimal decision functions. See Jullien [10] for a general exposition.

This paper develops an alternative approach to optimal bunching. We build on an insight due to Goldman, Leland and Sibley [8], namely that it is possible to view the principal's problem as choosing an optimal type assignment (mapping decisions into types) rather than as choosing an optimal decision function (mapping types into decisions). ${ }^{2}$ Incentive compatibility imposes a monotonicity constraint on type assignments, but the conditions under which this constraint is binding are different from those under which the monotonicity constraint on decision functions is binding. In particular, we show that the monotonicity constraint on type assignments can be ignored whenever two simple conditions are satisfied. The first requires the agent's utility function (net of any, possibly type dependent, reservation utility) to be quasi-convex in the agent's type. The second requires the virtual surplus function (i.e., surplus adjusted to account for the agent's informational rent (Myerson [18])) to be strictly concave. As we discuss in Section 4, these conditions are satisfied in many applications of the principal-agent model, including models of market making (cf. Biais, Martimort and Rochet [2]) and countervailing incentives

\footnotetext{
${ }^{2}$ See Wilson [24] for an extensive discussion of the assignment approach to optimal non-linear pricing and its relation to the standard approach.
} 
(cf. Lewis and Sappington $[12,13])$.

Because our assumptions ensure that the monotonicity constraint on type assignments is not binding, we can obtain optimal type assignments by point-wise maximization of the appropriate objective function. Optimal bunches correspond to the discontinuities of this point-wise solution and may arise for two reasons. First, the point-wise solution will be discontinuous if multiple types solve the maximization problem for a given decision. This possibility corresponds to the cases of optimal bunching discussed in Goldman, Leland and Sibley [8] and is the only possibility in models in which the agent's utility is monotonic in type (cf. Baron and Myerson [1], Mussa and Rosen [19]). Second, the point-wise solution is discontinuous if the agent's participation constraint is binding at an interior type (as in the models of market making and countervailing incentives mentioned above). In either case, our approach provides a simple characterization of the optimal bunch and also yields an alternative interpretation and derivation of the optimality conditions customarily obtained from the application of control theory.

The following section introduces the model. Section 3 presents a reformulation of the principal's problem (along the lines suggested by Goldman, Leland and Sibley [8]) that provides the starting point for our analysis. Section 4 shows how this problem can be solved by point-wise maximization. Section 5 concludes. Some of the more technical or lengthy (steps of) proofs are in the Appendix.

\section{The Model}

The principal and the agent contract on a one-dimensional decision $x \in[\underline{x}, \bar{x}] \equiv X \subset \mathbb{R}$ and a monetary transfer $m \in \mathbb{R}$. The agent's utility from trade depends on her type $\theta \in[\underline{\theta}, \bar{\theta}] \equiv \Theta \subset \mathbb{R}$ and is given by $u(x, \theta)-m$. The principal's utility from trade may also depend on the agent's type (i.e., we allow for common values) and is given by $v(x, \theta)+m$. The agent knows her type. From the principal's perspective the agent's type is drawn from the interval $\Theta$ according to the distribution function $F$, with differentiable density $f>0$. The functions $u$ and $v$ are assumed to be thrice continuously differentiable on $X \times \Theta$. In addition, we assume throughout that the 
agent's utility function satisfies the strict single-crossing property (denoting partial derivatives by subscripts):

$$
u_{x \theta}>0
$$

We formulate the principal's problem as the non-linear pricing problem of choosing a price function $t: X \rightarrow \mathbb{R}$ and an associated decision function $q: \Theta \rightarrow X$, to solve: ${ }^{3}$

$$
\max _{t, q} \int_{\underline{\theta}}^{\bar{\theta}}[v(q(\theta), \theta)+t(q(\theta))] f(\theta) d \theta
$$

subject to the incentive constraints

$$
q(\theta) \in \arg \max _{x}[u(x, \theta)-t(x)], \forall \theta \in \Theta
$$

and the participation constraints ${ }^{4}$

$$
\max _{x}[u(x, \theta)-t(x)] \geq 0, \forall \theta \in \Theta .
$$

A solution $(t, q)$ to this non-linear pricing problem has an optimal bunch $\left[\theta_{1}, \theta_{2}\right]$ at $x$ if $\theta_{1}<$ $\theta_{2}$ and $q(\theta)=x$ holds for all types in $\left(\theta_{1}, \theta_{2}\right)$. We are interested in obtaining a characterization of such optimal bunches from the solution to unconstrained maximization problems. To provide a more convenient starting point for this enterprize we reformulate the principal's problem.

\footnotetext{
${ }^{3}$ From the taxation principle (e.g., Rochet [20]), the nonlinear pricing problem we consider is equivalent to the familiar formulation in which the principal chooses a deterministic direct mechanism. Our assumptions (cf. Section 4) do not preclude the possibility that the principal might fare better with a stochastic mechanism (see Jullien [10] and Strausz [23]), replacing the set of decisions $X$ in the deterministic mechanism with the set of lotteries over $X$. It is not obvious how our reformulation of the principal's problem in the following section could be extended to deal with stochastic direct mechanisms, as there is no natural counterpart of the condition that type assignments be increasing. We restrict attention to the deterministic case throughout.

${ }^{4}$ We normalize the reservation utility of every type of agent to zero. This sacrifices no generality, as we can always interpret utilities as surpluses over a (possibly type dependent) reservation utility.
} 


\section{A Reformulation}

As noted by Goldman, Leland and Sibley [8] for a special case of the non-linear pricing problem introduced above, there is no loss of generality in restricting attention to price functions $t$ satisfying $^{5}$

$$
t(x)=t(\underline{x})+\int_{\underline{x}}^{x} u_{x}(\tilde{x}, \psi(\tilde{x})) d \tilde{x}, \quad \psi: X \rightarrow \Theta \text { increasing }
$$

when studying the principal's problem:

Lemma 1 Let $(t, q)$ solve (2) - (4). Then there exists a price function $t^{\prime}$ satisfying (5) such that $\left(t^{\prime}, q\right)$ solves (2) - (4).

Proof: Because $(t, q)$ satisfies (3) the associated rent function $r: \Theta \rightarrow \mathbb{R}$ given by

$$
r(\theta)=\max _{x}[u(x, \theta)-t(x)]
$$

is well-defined. Define $t^{\prime}: X \rightarrow \mathbb{R}$ by

$$
t^{\prime}(x)=\max _{\theta}[u(x, \theta)-r(\theta)]
$$

so that $t^{\prime}$ is the lower envelope of the price functions for which (6) holds. We will first show that $\left(t^{\prime}, q\right)$ solves (2) - (4) and then argue that it satisfies (5).

The following relationships (corresponding to the Fenchel inequalities from convex analysis, cf. Rockafellar [22, Section 12]) are immediate from (6) and (7):

$$
\begin{aligned}
t(x)+r(\theta) & \geq u(x, \theta), \forall(x, \theta) \\
r(\theta)+t^{\prime}(x) & \geq u(x, \theta), \forall(x, \theta) .
\end{aligned}
$$

From (8) we have $t(x) \geq \max _{\theta}[u(x, \theta)-r(\theta)]$ and thus $t(x) \geq t^{\prime}(x)$, implying

$$
u(q(\theta), q)-t^{\prime}(q(\theta)) \geq u(q(\theta), \theta)-t(q(\theta))=r(\theta),
$$

\footnotetext{
${ }^{5}$ We say that a function $\psi: X \rightarrow \Theta$ is increasing if $x_{1}>x_{2}$ implies $\psi\left(x_{1}\right) \geq \psi\left(x_{2}\right)$.
} 
and thus $r(\theta) \leq \max _{x}\left[u(x, \theta)-t^{\prime}(x)\right]$. From (9) we have $r(\theta) \geq \max _{x}\left[u(x, \theta)-t^{\prime}(x)\right]$, and hence, combining inequalities,

$$
r(\theta)=\max _{x}\left[u(x, \theta)-t^{\prime}(x)\right]
$$

This implies that $\left(t^{\prime}, q\right)$ satisfies (4) and, using (10), satisfies (3). Because $v(q(\theta), \theta)+t(q(\theta))=$ $v(q(\theta), \theta)+u(q(\theta), \theta)-r(\theta)$, the principal's profit depends on the price function only through $q$ and $r$, and it then follows that $\left(t^{\prime}, q\right)$ is optimal.

Applying Theorem 2 (in conjunction with their footnote 10) from Milgrom and Segal [17] to $t^{\prime}$ as defined by (7) yields the integral representation in (5), with the single crossing property (1) implying that $\psi$ is increasing (cf. Proposition 1 in Rochet [21]).

Throughout the following we will refer to an increasing $\psi$ as a type assignment and say that the pair $(t, \psi)$ is consistent if (5) holds. The advantage of restricting the non-linear pricing problem to price functions satisfying (5) lies in the fact that for such price functions there is a simple characterization of the associated decision functions $q$ satisfying (3). For every type assignment $\psi$ let

$$
\Psi(x)=\left[\lim _{y \uparrow x} \psi(y), \lim _{y \downarrow x} \psi(y)\right]
$$

where we adopt the conventions

$$
\lim _{y \uparrow \underline{x}} \psi(y)=\underline{\theta}, \lim _{y \downarrow \bar{x}} \psi(y)=\bar{\theta} .
$$

For any consistent $(t, \psi)$, the corresponding decision functions $q$ satisfying the incentive constraints (3) can be obtained by taking a selection from the inverse of the increasing correspondence $\Psi$. In particular, it is optimal for type $\theta=\psi(x)$ to choose decision $x$ when faced with the price schedule $t$.

Lemma 2 Let $(t, \psi)$ be consistent. Then

$$
x \in \arg \max _{\breve{x}}\{u(\breve{x}, \theta)-t(\breve{x})\} \Leftrightarrow \theta \in \Psi(x) .
$$


Proof: We have $x \in \arg \max _{\breve{x}}[u(\breve{x}, \theta)-t(\breve{x})]$ if and only if $u(x, \theta)-u(\breve{x}, \theta) \geq t(x)-t(\breve{x})$ for all $\breve{x}$, which from (5) is equivalent to

$$
\int_{\breve{x}}^{x} u_{x}(\tilde{x}, \theta) d \tilde{x} \geq \int_{\breve{x}}^{x} u_{x}(\tilde{x}, \psi(\tilde{x})) d \tilde{x}
$$

From (1) and the fact that $\psi$ is increasing, this condition holds for all $\breve{x}$ if and only if $\theta \in \Psi(x)$.

The inverse relationship established in Lemma 2 can be exploited to eliminate the decision function from the principal's objective $(2)$. For consistent $(t, \psi)$ let

$$
G(t, \psi)=\int_{\underline{x}}^{\bar{x}} s(x, \psi(x)) d x+V(\underline{x})+t(\underline{x}),
$$

where

$$
V(x)=\int_{\underline{\theta}}^{\bar{\theta}} v(x, \theta) f(\theta) d \theta
$$

and

$$
s(x, \theta)=\int_{\theta}^{\bar{\theta}}\left[u_{x}(x, \theta)+v_{x}(x, \tilde{\theta})\right] f(\tilde{\theta}) d \tilde{\theta}
$$

Lemma 3 Let $(t, \psi)$ be consistent and let $q$ satisfy (3). Then

$$
\int_{\underline{\theta}}^{\bar{\theta}}[v(q(\theta), \theta)+t(q(\theta))] f(\theta) d \theta=G(t, \psi) .
$$

Proof: Using (5) we can rewrite the principal's payoff as

$$
\int_{\underline{\theta}}^{\bar{\theta}}[v(q(\theta), \theta)+t(q(\theta))] f(\theta) d \theta=\int_{\underline{\theta}}^{\bar{\theta}} \int_{\underline{x}}^{q(\theta)}\left[v_{x}(x, \theta)+u_{x}(x, \psi(x))\right] f(\theta) d x d \theta+V(\underline{x})+t(\underline{x}) .
$$

Because of the inverse relationship between $q$ and $\psi$ (Lemma 2), we can apply Fubini's theorem to the double integral to obtain

$$
\int_{\underline{\theta}}^{\bar{\theta}} \int_{\underline{x}}^{q(\theta)}\left[v_{x}(x, \theta)+u_{x}(x, \psi(x))\right] f(\theta) d x d \theta=\int_{\underline{x}}^{\bar{x}} \int_{\psi(x)}^{\bar{\theta}}\left[v_{x}(x, \theta)+u_{x}(x, \psi(x))\right] f(\theta) d \theta d x .
$$

To see the economic intuition behind (14)-(16), note that the term $V(\underline{x})+t(\underline{x})$ corresponds to the principal's payoff from the contract in which every type of the agent takes the decision $\underline{x}$ 
in return for the transfer $t(\underline{x})$. The integral appearing in (14) takes into account the additional profits that result from providing further marginal units of $x$ to the appropriate types of agents. In particular, we can think of $u_{x}(x, \psi(x))$ as the price charged for the $x$-th marginal unit, with this marginal unit being provided (due to (1) and (13)) to all types $\tilde{\theta}$ higher than $\psi(x)$, resulting in a revenue of $u_{x}(x, \psi(x))(1-F(\psi(x)))$ for the principal. The principal's cost of providing the $x$-th marginal unit to type $\tilde{\theta}$ is given by $-v_{x}(x, \tilde{\theta})$, yielding $s(x, \psi(x))$ as the profit from providing the $x$-th marginal unit.

The importance of Lemma 3 is that we may reformulate the principal's problem, as given by (2) - (4), by first adding (5) to the constraints and then eliminating the decision function $q$. The resulting program is

$$
\max _{\psi, t} \int_{\underline{x}}^{\bar{x}} s(x, \psi(x)) d x+V(\underline{x})+t(\underline{x})
$$

subject to

$$
t(x)=t(\underline{x})+\int_{\underline{x}}^{x} u_{x}(\tilde{x}, \psi(\tilde{x})) d \tilde{x}, \quad \psi \text { increasing }
$$

and

$$
\max _{x}[u(x, \theta)-t(x)] \geq 0, \forall \theta \in \Theta .
$$

In the remainder of the paper we study the problem (17) - (19). In doing so we will refer to a price function as feasible if it satisfies (18) - (19). A consistent $(t, \psi)$ is optimal if it solves (17) - (19).

Throughout the following we will identify type assignments that agree almost everywhere, thus writing $\psi=\psi^{\prime}$ whenever $\psi(x)=\psi^{\prime}(x)$ holds for almost all $x \in X .{ }^{6}$ Finally, note that if $(t, \psi)$ is optimal, it follows from Lemma 2 that a decision function $q$ satisfying (3) has an optimal bunch at $x$ if and only if $\Psi(x)$ is non-singleton, with the optimal bunch given by $\Psi(x)$.

\footnotetext{
${ }^{6} \mathrm{As}$ a result, if $(t, \psi)$ is consistent then $\left(t, \psi^{\prime}\right)$ is consistent if and only if $\psi=\psi^{\prime}$. Our identification eliminates spurious non-uniqueness issues which arise solely from the fact that the same price function $t$ (which determines the principal's payoff) may be consistent with type assignments that are equal in the sense just defined but do not agree for all $x$.
} 


\section{Point-wise Maximization}

In this section, culminating in Propositions 1 - 3, we show that two conditions suffice to determine optimal type assignments, and thus (by Lemma 2) optimal decision functions, by solving a collection of unconstrained maximization problems.

\subsection{Assumptions}

The first condition we require is

Assumption 1 The agent's utility function $u$ is quasi-convex in $\theta$ for all $x$.

Assumption 1 is (trivially) satisfied in standard applications of the principal-agent model in which the agent's utility is increasing in $\theta$ for all $x$ (cf. Goldman, Leland, and Sibley [8], Maskin and Laffont [15], and Mussa and Rosen [19]) or decreasing in $\theta$ for all $x$ (cf. Baron and Myerson [1]; see also Laffont and Tirole [11]). It also holds in models of (monopoly) market making, as in Biais, Martimort and Rochet [2] (see also Glosten [6, 7]), in which $0 \in(\underline{x}, \bar{x})$ corresponds to the no-trade outcome satisfying $u(0, \theta)=0$ for all $\theta .^{7}$ Assumption 1 also holds in models with countervailing incentives in which the agent's reservation utility profile is concave (cf. Maggi and Rodriguez-Clare [14], who extend Lewis and Sappington [12, 13], and Feenstra and Lewis [4]). With their analysis of the case in which the agent's reservation profile is strictly convex, Maggi and Rodriguez-Clare [14] also provide an example of a model violating Assumption 1. Further examples of models violating Assumption 1 are presented and analyzed in Jullien [10]. ${ }^{8}$ Note that Assumption 1 does not preclude optimal bunching. Indeed, the models of monopoly market making and countervailing incentives cited above are among the prime examples of models in which bunches are an essential feature of the solution to the principal's problem.

\footnotetext{
${ }^{7}$ The single crossing property (1) then ensures that $u(x, \theta)$ is decreasing in $\theta$ for all $x<0$ and increasing in $\theta$ for all $x>0$, implying Assumption 1 .

${ }^{8}$ In particular, any model satisfying Jullien's assumption of homogeneity for a non-constant quantity profile violates Assumption 1.
} 
To formulate our second condition let

$$
\begin{aligned}
\sigma(x, \theta) & =v(x, \theta)+u(x, \theta)-\frac{1-F(\theta)}{f(\theta)} u_{\theta}(x, \theta) \\
\beta(x, \theta) & =v(x, \theta)+u(x, \theta)+\frac{F(\theta)}{f(\theta)} u_{\theta}(x, \theta) .
\end{aligned}
$$

The functions $\sigma: X \times \Theta \rightarrow \mathbb{R}$ and $\beta: X \times \Theta \rightarrow \mathbb{R}$ are the virtual surplus functions (Myerson [18]), familiar from the standard approach to the principal-agent problem. ${ }^{9}$

Assumption 2 The virtual surplus functions $\sigma$ and $\beta$ satisfy $\sigma_{x x}<0$ and $\beta_{x x}<0$, and hence are strictly concave in $x$ for all $\theta$.

Assumption 2 is commonly encountered in the analysis of principal-agent models, where its role is to ensure uniqueness (and thus also continuity) of the decision functions $q^{\sigma}$ and $q^{\beta}$ that are obtained from the point-wise maximization of $\sigma(x, \theta)$ and $\beta(x, \theta)$ with respect to $x$. Assumption 2 also implies that $u(x, \theta)+v(x, \theta)$ is strictly concave in $x$ for all $\theta$, ensuring that the decision function $q^{F B}$ which results from the point-wise maximization of the first-best surplus, $q^{F B}(\theta)=\arg \max _{x} u(x, \theta)+v(x, \theta)$, is uniquely defined and continuous. ${ }^{10}$ Note, however, that $q^{F B}$ need not be increasing, as our assumptions impose no restriction on $v_{x \theta}(x, \theta)$. Consequently, the model may fail to be responsive (see Guesnerie and Laffont [9]). Even in the simplest cases in which $u$ is either increasing in $\theta$ for all $x$ or decreasing in $\theta$ for all $x$, Assumption 2 does not preclude the occurrence of optimal bunches. ${ }^{11}$

Theorems 3 and 4 in Jullien [10] provide a complete characterization of the solution to the principal's problem under Assumption 2. Our analysis provides an alternative derivation

\footnotetext{
${ }^{9}$ The value $\sigma(x, \theta)$ is the surplus achieved by allocating quantity $x$ to type $\theta$, taking into account the rents that must then be left to types higher than $\theta$ if this is to be incentive compatible. Similarly, $\beta(x, \theta)$ can be viewed as the surplus from allocating quantity $x$ to type $\theta$, taking into account the effect on the rents of types below $\theta$.

${ }^{10}$ Conversely, if $u(x, \theta)+v(x, \theta)$ is strictly concave in $x$ and $u_{x x \theta}=0$, as is commonly the case, then Assumption 2 holds.

${ }^{11}$ To preclude bunching, $q^{\sigma}$ (resp. $q^{\beta}$ ) must be increasing, as would be guaranteed by the assumption $\sigma_{x \theta}>0$ (resp. $\beta_{x \theta}>0$ ), which we do not impose.
} 
and characterization which, under Assumptions 1 and 2, dispenses with the optimal control techniques that are at the heart of Jullien's derivation.

\subsection{Adapted Price Functions}

As a first step in our analysis we demonstrate that Assumption 1 yields a simple characterization of feasible price functions. In particular, feasible price functions have the property that there is some decision $\hat{x}$ such that all types of the agent obtain at least their reservation utility if they choose $\hat{x}$ and pay $t(\hat{x})$. We refer to such price schedules as being adapted.

Definition 1 Let $\hat{x} \in X$. A price function $t$ is $\hat{x}$-adapted if

$$
t(\hat{x}) \leq u_{\min }(\hat{x}) \equiv \min _{\theta} u(\hat{x}, \theta)
$$

It is adapted if it is $\hat{x}$-adapted for some $\hat{x}$.

Note that every adapted price function satisfies (19). Hence, every $t$ that satisfies (18) and is adapted is feasible. The following lemma establishes the converse. Let

$$
\Theta_{\min }(x)=\arg \min _{\theta} u(x, \theta)
$$

Lemma 4 Let Assumption 1 hold. Then every feasible price function is adapted.

Proof: Let $t$ be feasible and let $\psi$ be a type assignment such that $(t, \psi)$ is consistent. Standard fixed-point arguments (see the Appendix) imply that there exists $\hat{x} \in X$ such that $\Psi(\hat{x}) \cap$ $\Theta_{\min }(\hat{x}) \neq \emptyset$. Let $\hat{\theta} \in \Psi(\hat{x}) \cap \Theta_{\min }(\hat{x})$. Because $\hat{\theta} \in \Psi(\hat{x})$ and $t$ is feasible, we have $\hat{x} \in$ $\arg \max _{x}[u(x, \hat{\theta})-t(x)] \geq 0$, which implies

$$
u(\hat{x}, \hat{\theta}) \geq t(\hat{x})
$$

Because $\hat{\theta} \in \Theta_{\min }(\hat{x})$, this inequality implies $u_{\min }(\hat{x}) \geq t(\hat{x})$. Hence, $t$ is $\hat{x}$-adapted.

Assumption 1 thus implies that we may replace the participation constraint (19) with the constraint that (22) holds for some $\hat{x} \in X$. This suggests a simple two-stage procedure for solving 
(17) - (19): in the first stage, maximize (17) subject to (18) and the additional constraint that the price function be $\hat{x}$-adapted. In the second stage, maximize with respect to $\hat{x}$ to obtain the solution to the principal's problem. To pursue this procedure we find it convenient to offer:

Definition $2 A$ pair $(t, \psi)$ is $\hat{x}$-optimal if it maximizes (17) subject to (18) and (22).

Remark 1. The second stage of the maximization procedure described above is not needed if there exists $\hat{x}$ such that all feasible price functions are $\hat{x}$-adapted, implying that an $\hat{x}$-optimal $(t, \psi)$ solves the program $(17)-(19)$. For instance, if the agent's utility function is increasing in $\theta$ for all $x$, then every feasible price function is $\underline{x}$-adapted (because every type assignment $\psi$ satisfies $\underline{\theta} \in \Psi(\underline{x})$ (cf. (12)) and $\underline{\theta} \in \Theta_{\min }(\underline{x})$ (cf. (23))). An analogous argument shows that every feasible price schedule is $\bar{x}$-adapted if the agent's utility function is decreasing in $\theta$ for all $x$. If $u(0, \theta)=0$ for all $\theta$, as in a model of market making (see the discussion following Assumption 1), then every feasible price function is adapted at 0. See Remark 2 (below) for further discussion.

\subsection{The First Step: $\hat{x}$-optimality}

To characterize $\hat{x}$-optimal $(t, \psi)$, define $b: X \times \Theta \rightarrow \mathbb{R}$ by

$$
b(x, \theta)=s(x, \theta)-V_{x}(x)-u_{x}(x, \theta)
$$

Using the definitions of $s$ and $V$ given by (15)-(16) and rearranging yields

$$
b(x, \theta)=-\int_{\underline{\theta}}^{\theta}\left[u_{x}(x, \theta)+v_{x}(x, \tilde{\theta})\right] f(\tilde{\theta}) d \tilde{\theta} .
$$

Equation (25) provides an interpretation of $b$ analogous to the interpretation of $s$ offered in Section 3: $b(x, \theta)$ represents the principal's payoff from obtaining the $x$-th marginal unit from all types lower than $\theta$ at the price $u_{x}(x, \theta)$. Condition (5) and definition (24) allow us to rewrite the principal's payoff (defined in (14)), for any $\hat{x} \in X$ and any consistent $(t, \psi)$, as

$$
G(t, \psi)=\int_{\underline{x}}^{\hat{x}} b(x, \psi(x)) d x+\int_{\hat{x}}^{\bar{x}} s(x, \psi(x)) d x+V(\hat{x})+t(\hat{x}) .
$$


It is then immediate that every $\hat{x}$-optimal $(t, \psi)$ must satisfy $(22)$ with equality. We may thus eliminate the price function from the maximization problem to obtain:

Lemma 5 A consistent $\left(t^{*}, \psi^{*}\right)$ is $\hat{x}$-optimal if and only if $t^{*}(\hat{x})=u_{\min }(\hat{x})$ and $\psi^{*}$ solves

$$
\max _{\psi \text { increasing }} \int_{\underline{x}}^{\hat{x}} b(x, \psi(x)) d x+\int_{\hat{x}}^{\bar{x}} s(x, \psi(x)) d x .
$$

To identify $\hat{x}$-optimal $(t, \psi)$ it remains to solve (27). Assumption 2 dispenses with the monotonicity constraint by ensuring that the correspondences defined by

$$
\Upsilon^{b}(x)=\arg \max _{\theta} b(x, \theta), \quad \Upsilon^{s}(x)=\arg \max _{\theta} s(x, \theta)
$$

are increasing, i.e., every selection from these correspondences is increasing and is thus a type assignment. Let

$$
\phi^{b}(x)=\min \Upsilon^{b}(x), \quad \phi^{s}(x)=\max \Upsilon^{s}(x)
$$

Lemma 6 Let Assumption 2 hold. Then $\phi^{b}$ and $\phi^{s}$ are increasing. Furthermore, if $\psi$ is a selection from $\Upsilon^{b}$ (resp. from $\Upsilon^{s}$ ) then $\psi=\phi^{b}\left(\right.$ resp. $\left.\psi=\phi^{s}\right) .{ }^{12}$

Proof: Berge's maximum theorem ([3, Theorem 12.1]) implies that $\Upsilon^{b}$ and $\Upsilon^{s}$ are compact, ensuring that $\phi^{b}$ and $\phi^{s}$ are well-defined. If the correspondences $\Upsilon^{b}$ and $\Upsilon^{s}$ are increasing, they must be single-valued for almost all $x \in X$, implying that every other selection from $\Upsilon^{b}$ (resp. from $\Upsilon^{s}$ ) is an increasing type assignment equal to $\phi^{b}$ (resp. $\phi^{s}$ ). It remains to show both $\Upsilon^{b}$ and $\Upsilon^{s}$ are increasing. From Theorem 4 in Milgrom and Shannon [16], a sufficient condition for this is that for all $(x, \theta)$,

$$
\begin{aligned}
& b_{x \theta}(x, \theta)=-\left[u_{x x}(x, \theta)+v_{x x}(x, \theta)\right] f(\theta)-F(\theta) u_{x x \theta}(x, \theta)>0, \\
& s_{x \theta}(x, \theta)=-\left[u_{x x}(x, \theta)+v_{x x}(x, \theta)\right] f(\theta)+[1-F(\theta)] u_{x x \theta}(x, \theta)>0 .
\end{aligned}
$$

A straightforward calculation, using (21), gives $b_{x \theta}(x, \theta)=-\beta_{x x}(x, \theta) f(\theta)$, so that condition (30) is equivalent to $\beta_{x x}<0$. Similarly, from $(20), s_{x \theta}(x, \theta)=-\sigma_{x x}(x, \theta) f(\theta)$, so that condition (31) is equivalent to $\sigma_{x x}<0$. The result then follows from Assumption 2.

\footnotetext{
${ }^{12}$ Recall that we write $\psi=\psi^{\prime}$ if $\psi$ and $\psi^{\prime}$ agree almost everywhere.
} 
For the cases $\hat{x}=\underline{x}$ and $\hat{x}=\bar{x}$ it is immediate from Lemma 6 that a type assignment $\psi$ is $\hat{x}$-optimal if and only $\psi=\phi^{s}$, respectively $\psi=\phi^{b}$, and can thus be obtained from the point-wise maximization of the objective function in $(27) \cdot{ }^{13}$ For the case $\hat{x} \in(\underline{x}, \bar{x})$, an additional argument is needed to ensure that point-wise maximization does not violate the monotonicity constraint by inducing a downward discontinuity at $\hat{x}$. Using (1) and (24), we have

$$
s_{\theta}(x, \theta)>b_{\theta}(x, \theta), \forall(x, \theta),
$$

and thus

$$
\phi^{b}(x) \leq \phi^{s}(x), \forall x \in X
$$

ensuring that such a downward discontinuity cannot arise. Consequently, as we record in the following lemma, an $\hat{x}$-optimal type assignment is uniquely determined by pasting $\phi^{b}$ and $\phi^{s}$ at $\hat{x}$.

Lemma 7 Let Assumption 2 hold. Then a type assignment $\psi$ is $\hat{x}$-optimal if and only if $\psi=\phi^{\hat{x}}$, where

$$
\phi^{\hat{x}}(x)= \begin{cases}\phi^{b}(x), & \text { if } x \leq \hat{x} \\ \phi^{s}(x), & \text { if } x>\hat{x}\end{cases}
$$

Remark 2 For those cases in which there exists $\hat{x}$ such that all feasible price schedules are $\hat{x}$-adapted (see Remark 1), Lemma 7 finishes our task of obtaining the solution to the principal's problem from a point-wise maximization. To illustrate with a simple but non-trivial example, consider a special case of the monopoly screening problem from Biais, Martimort and Rochet [2], in which $\underline{x}<0<\bar{x}$, the agent's type is distributed uniformly on $[\underline{\theta}, \bar{\theta}]$, satisfying $\underline{\theta}<0<\bar{\theta}$, and utility functions are given by $u(x, \theta)=x \theta-\gamma x^{2} / 2$ and $v(x, \theta)=-\alpha x \theta$ with $\alpha \in(0,1)$ and $\gamma>0$. We have noted that Assumption 1 holds and that every feasible price function is 0-adapted. Assumption 2 also holds. Thus, $\phi^{0}$ is the optimal type assignment. Solving the

\footnotetext{
${ }^{13}$ In addition, it is clear that assuming only $\sigma_{x x}<0$ (only $\beta_{x x}<0$ ) suffices to obtain Lemma 6 for the case $\hat{x}=\underline{x}(\hat{x}=\bar{x})$.
} 
maximization problems defining $\phi^{b}$ and $\phi^{s}$ yields the optimal type assignment

$$
\phi^{0}(x)= \begin{cases}\max \left\{\frac{\underline{\theta}+\gamma x}{2-\alpha}, \underline{\theta}\right\}, & \text { if } x \leq 0 \\ \min \left\{\frac{\bar{\theta}+\gamma x}{2-\alpha}, \bar{\theta}\right\}, & \text { if } x>0\end{cases}
$$

Note that there is an optimal bunch at zero given by $[\underline{\theta} /(2-\alpha), \bar{\theta} /(2-\alpha)]$.

\subsection{The Second Step: Where to Adapt}

Turning to the second step of the maximization procedure outlined above, let $W: X \rightarrow \mathbb{R}$ denote the value function of the maximization problem defining $\hat{x}$-optimality. From Lemma 5 and equation (26), this is given by

$$
W(\hat{x})=\max _{\psi \text { increasing }}\left\{\int_{\underline{x}}^{\hat{x}} b(x, \psi(x)) d x+\int_{\hat{x}}^{\bar{x}} s(x, \psi(x)) d x\right\}+V(\hat{x})+u_{\min }(\hat{x}) .
$$

As we have already argued, Assumption 1 implies that $(t, \psi)$ is optimal if and only if it is $x^{*}$-optimal for $x^{*} \in \arg \max _{\hat{x}} W(\hat{x})$. Combining this observation with Lemmas 5 and 7 yields the following:

Proposition 1 Let Assumptions 1 and 2 hold. Then a consistent $(t, \psi)$ is optimal if and only if there exists $x^{*} \in \arg \max _{\hat{x}} W(\hat{x})$ such that $\psi=\psi^{x^{*}}$ and $t\left(x^{*}\right)=u_{\min }\left(x^{*}\right)$.

Proposition 1 ensures that the principal's problem can be solved by point-wise maximization. However, it would be desirable to have a more explicit characterization of the condition $x^{*} \in \arg \max _{\hat{x}} W(\hat{x})$. The following result (proven in the Appendix) provides the appropriate first order condition. Recall, from $(23), \Theta_{\min }(x)=\arg \min _{\theta} u(x, \theta)$.

Proposition 2 Let Assumptions 1 and 2 hold and let $H: X \times \Theta \rightarrow \mathbb{R}$ be defined by

$$
H(\hat{x}, \hat{\theta})=\int_{\underline{x}}^{\hat{x}} b\left(x, \phi^{b}(x)\right) d x+\int_{\hat{x}}^{\bar{x}} s\left(x, \phi^{s}(x)\right) d x+V(\hat{x})+u(\hat{x}, \hat{\theta}) .
$$


Then $x^{*} \in \arg \max _{\hat{x}} W(\hat{x})$ holds if and only if there exists $\theta^{*} \in \Theta_{\min }\left(x^{*}\right)$ such that

$$
H_{\hat{x}}\left(x^{*}, \theta^{*}\right)\left\{\begin{array}{l}
\leq 0, \quad \text { if } x^{*}=\underline{x} \\
=0, \quad \text { if } x^{*} \in(\underline{x}, \bar{x}) \\
\geq 0, \quad \text { if } x^{*}=\bar{x} .
\end{array}\right.
$$

The existence of an $x^{*} \in \arg \max _{\hat{x}} W(\hat{x})$ and hence an optimal $\left(t^{*}, \psi^{*}\right)$ is immediate from the (absolute) continuity of the value function $W$ (cf. the proof of Proposition 2). Theorem 4 in Jullien [10] shows that the optimal $\left(t^{*}, \psi^{*}\right)$ is unique. ${ }^{14}$

\subsection{Optimal Bunches}

Proposition 1 and Lemma 2 imply that the solution to the principal's problem will have an optimal bunch at $x$ if and only if the the optimal type assignment $\phi^{x^{*}}$ given by (33) satisfies (cf. (11) and (12))

$$
\lim _{y \uparrow x} \phi^{x^{*}}(y)<\lim _{y \downarrow x} \phi^{x^{*}}(y) .
$$

Because $\phi^{b}$ and $\phi^{s}$ are selections from the upper-hemi-continuous argmax-correspondences $\Upsilon^{b}$ and $\Upsilon^{s}$ (cf. (28)) and (29), we immediately obtain an explicit characterization of optimal bunches in terms of the solutions of the point-wise maximization of $b$ and $s:{ }^{15}$

Proposition 3 Let Assumptions 1 and 2 hold and let $x^{*} \in \arg \max _{\hat{x}} W(\hat{x})$. Then there is an optimal bunch at $x \in X$ if and only if (37) holds. If there is an optimal bunch $\left[\theta_{1}(x), \theta_{2}(x)\right]$ at

\footnotetext{
${ }^{14}$ Alternatively, a straightforward (but tedious) extension of the proof of Proposition 2 establishes uniqueness by showing that, if $\arg \max _{\hat{x}} W(\hat{x})$ has multiple maximizers, then either the corresponding $x^{*}$-optimal type assignment is $\phi^{b}$ for all $x^{*} \in \arg \max _{\hat{x}} W(\hat{x})$ or is $\phi^{s}$ for all $x^{*} \in \arg \max _{\hat{x}} W(\hat{x})$.

${ }^{15}$ For the sake of clarity we state this characterization in (38) only for optimal bunches occurring at interior decisions. The characterization of optimal bunches at the boundaries is equally straightforward from our previous results, but requires a number of case distinctions and thus is somewhat cumbersome to state. A simple sufficient condition to rule out bunches at $\underline{x}$ and $\bar{x}$ is given by $\phi^{s}(\underline{x})=\underline{\theta}$ and $\phi^{b}(\bar{x})=\bar{\theta}$.
} 
$x \in(\underline{x}, \bar{x})$ it is given by

$$
\begin{aligned}
& \theta_{1}(x)=\min \Upsilon^{b}(x), \quad \theta_{2}(x)=\max \Upsilon^{b}(x), \quad \text { if } x<x^{*} \\
& \theta_{1}(x)=\min \Upsilon^{b}(x), \quad \theta_{2}(x)=\max \Upsilon^{s}(x), \quad \text { if } x=x^{*} \\
& \theta_{1}(x)=\min \Upsilon^{s}(x), \quad \theta_{2}(x)=\max \Upsilon^{s}(x), \quad \text { if } x>x^{*} .
\end{aligned}
$$

To relate this result to existing characterizations of optimal bunches resulting from the application of optimal control techniques, consider (first) bunches at $x \neq x^{*}$. It is immediate from (38) that such bunches are excluded if $b$ and $s$ are strictly quasi-concave in $\theta$ for all $x,{ }^{16}$ as in this case $\Upsilon^{b}$ and $\Upsilon^{s}$ are single-valued. Supposing that there is an optimal bunch at such an $x$, we easily recover the result that the average of the marginal virtual surpluses over a bunch must be equal to zero (see, for instance, Fudenberg and Tirole [5, Appendix to Chapter 7]):

Corollary 1 Let Assumptions 1 and 2 hold. If $\left[\theta_{1}, \theta_{2}\right]$ is an optimal bunch at $x \in\left(\underline{x}, x^{*}\right)$ then

$$
\int_{\theta_{1}}^{\theta_{2}} \beta_{x}(x, \theta) f(\theta) d \theta=0
$$

If $\left[\theta_{1}, \theta_{2}\right]$ is an optimal bunch at $x \in\left(x^{*}, \bar{x}\right)$ then

$$
\int_{\theta_{1}}^{\theta_{2}} \sigma_{x}(x, \theta) f(\theta) d \theta=0
$$

Proof: Consider the case $x \in\left(\underline{x}, x^{*}\right)$ (the other case is analogous). From the first line in (38), we have $b\left(x, \theta_{1}\right)=b\left(x, \theta_{2}\right)$ and thus $\int_{\theta_{1}}^{\theta_{2}} b_{\theta}(x, \theta) d \theta=0$. The result is then immediate from the identity $b_{\theta}(x, \theta)=-\beta_{x}(x, \theta) f(\theta)$.

Consider next the characterization of optimal bunches at $x^{*}$. If we exclude the trivial special cases in which either $\phi^{b}$ or $\phi^{s}$ is an optimal type assignment, ${ }^{17}$ then there must be an optimal

\footnotetext{
${ }^{16}$ This provides a simple alternative to the standard assumptions guaranteeing the monotonicity of $q^{\beta}$ and $q^{\sigma}$. See Section 2.1 in Jullien [10].

${ }^{17}$ These cases can only occur if an optimal decision function $q$ has the property that either $q(\theta) \geq x^{*}$ holds for all $\theta$ or $q(\theta) \leq x^{*}$ holds for all $\theta$. A simple sufficient condition ensuring that neither $\phi^{b}$ nor $\phi^{s}$ is optimal is that the first-best decision function (cf. Section 4.1) $q^{F B}$ satisfies $q^{F B}(\underline{\theta}) \leq x^{*} \leq q^{F B}(\bar{\theta})$.
} 
bunch at $x^{*}$. Furthermore, such a bunch again satisfies (the appropriate generalization of) the condition that the average marginal virtual surplus over the bunch must be equal to zero (cf. Maggi and Rodriguez-Clare [14, Lemma 5]):

Corollary 2 Let Assumptions 1 and 2 hold and suppose $\phi^{x^{*}} \neq \phi^{s}$ and $\phi^{x^{*}} \neq \phi^{b}$. Then $x^{*} \in$ $(\underline{x}, \bar{x})$ and there is an optimal bunch $\left[\theta_{1}, \theta_{2}\right]$ at $x^{*}$. This optimal bunch satisfies

$$
\int_{\theta_{1}}^{\theta^{*}} \beta_{x}\left(x^{*}, \theta\right) f(\theta) d \theta+\int_{\theta^{*}}^{\theta_{2}} \sigma_{x}\left(x^{*}, \theta\right) f(\theta) d \theta=0
$$

for some $\theta^{*} \in \arg \min _{\theta} u\left(x^{*}, \theta\right)$.

Proof: $x^{*} \in(\underline{x}, \bar{x})$ is immediate from the assumption $\phi^{x^{*}} \neq \phi^{s}$ and $\phi^{x^{*}} \neq \phi^{b}$. To show that there must be an optimal bunch at $x^{*}$ it suffices to show that $\phi^{b}\left(x^{*}\right)<\phi^{s}\left(x^{*}\right)$. From (32), this must be the case unless $\phi^{b}\left(x^{*}\right)=\phi^{s}\left(x^{*}\right)=\underline{\theta}$ or $\phi^{b}\left(x^{*}\right)=\phi^{s}\left(x^{*}\right)=\bar{\theta}$. Because $\phi^{b}$ and $\phi^{s}$ are increasing, in the first of these cases we have $\phi^{x^{*}}=\phi^{s}$, while in the second we have $\phi^{x^{*}}=\phi^{b}$. In either case we have a contradiction to the assumption $\phi^{x^{*}} \neq \phi^{s}$ and $\phi^{x^{*}} \neq \phi^{b}$. Hence, there is an optimal bunch at $x^{*}$, satisfying $\theta_{1}=\phi^{b}\left(x^{*}\right)$ and $\theta_{2}=\phi^{s}\left(x^{*}\right)$ (from the second line of (36) and (29)). Because $x^{*}$ is interior, (36) implies that there exists $\theta^{*} \in \Theta_{\min }\left(x^{*}\right)$ such that $H_{x}\left(x^{*}, \theta^{*}\right)=0$. From (42) (in the proof of Proposition 2), this is equivalent to $\left[b\left(x^{*}, \theta_{1}\right)-\right.$ $\left.b\left(x^{*}, \theta^{*}\right)\right]-\left[s\left(x^{*}, \theta_{2}\right)-s\left(x^{*}, \theta^{*}\right)\right]=0$. Using the identities $b_{\theta}(x, \theta)=-\beta_{x}(x, \theta) f(\theta)$ and $s_{\theta}(x, \theta)=$ $-\sigma_{x}(x, \theta) f(\theta) d \theta$ and integrating by parts yields $\left[b\left(x^{*}, \theta_{1}\right)-b\left(x^{*}, \theta^{*}\right)\right]=\int_{\theta_{1}}^{\theta^{*}} \beta_{x}\left(x^{*}, \theta\right) f(\theta) d \theta$ and $\left[s\left(x^{*}, \theta_{2}\right)-s\left(x^{*}, \theta^{*}\right)\right]=-\int_{\theta^{*}}^{\theta_{2}} \sigma_{x}\left(x^{*}, \theta\right) f(\theta) d \theta$ and thus the result.

\section{Conclusion}

We have identified a class of principal-agent models in which a solution can be obtained from a collection of unconstrained point-wise maximization problems. This characterization of optimal type assignments has its limitations. It does not apply, for example, in cases where the agent's participation constraint binds at multiple, isolated types (see Maggi and Rodriguez-Claire [14] and Jullien [10] for examples where this is the case). However, it covers a wide variety of common cases. 
We see two promising possibilities for extending our analysis. First, as long as the agent's utility function is quasi-convex, Lemma 5 characterizes $\hat{x}$-optimal pairs $(t, \psi)$. Hence, even without strictly concave virtual surplus functions, the methods presented here allow a significant simplification of the participation constraint.

Second, our approach provides an alternative perspective on the comparative statics of the principal's problem. If Assumptions 1 and 2 hold, then the solution to the principal's problem is determined by the point-wise solutions $\phi^{b}$ and $\phi^{s}$ and the value $x^{*}$ at which they are pasted. The effects of changes in the underlying parameters can thus be inferred from their effect on $\phi^{b}, \phi^{s}$, and $x^{*}$. For example, consider replacing the utility function $u(x, \theta)$ with the function $u(x, \theta)-\tilde{u}(\theta)$, for some decreasing function $\tilde{u}$. This corresponds to a type-dependent increase in the agent's reservation values. The implications are clear from Propositions 1 and 2. Since $s$ and $b$ do not depend on the agent's reservation value, this change can affect the optimal assignment only through $x^{*}$. Since $\tilde{u}$ is decreasing, the set $\arg \min _{\theta} u(x, \theta)$ in Proposition 2 must increase, which in turn increases $H_{\hat{x}}(x, \theta)$. This ensures that $x^{*}$ must increase. As a result the optimal decision function decreases, as the jump from $\phi^{b}$ to $\phi^{s}$ now optimally occurs at a larger decision. We anticipate developing more such implications in future work.

\section{Appendix}

Proof of Lemma 4, Details: Define the correspondence $L: X \rightarrow \mathbb{R}$ by

$$
L(x)=\left\{\theta-\theta^{\prime}: \theta \in \Psi(x), \theta^{\prime} \in \Theta_{\min }(x)\right\} .
$$

We have to show that there exists $x \in X$ such that $0 \in L(x)$.

The correspondence $L$ is convex-valued (because $\Psi(x)$ is convex and the quasiconvexity of $u(x, \theta)$ ensures that $\Theta_{\min }(x)$ is convex), upper hemicontinuous and compact (because $\Psi$ is upper hemicontinuous and compact-valued and, by Berge's maximum theorem ([3, Theorem 12.1]), so is $\Theta_{\text {min }}$ ). In addition, $\min L(\underline{x}) \leq 0$ (because $\underline{\theta} \in \Psi(\underline{x})$, from (12)) and $\max L(\bar{x}) \geq 0$ (because $\bar{\theta} \in \Psi(\bar{x})$, from (12)). Let $\max L(\underline{x})<0$ and $\min L(\bar{x})>0$, since otherwise we immediately have 
$0 \in L(\underline{x})$ or $0 \in L(\bar{x})$. Then the correspondence $J$ defined on $[\underline{x}-1, \bar{x}+1]$ by

$$
J(x)=\left\{x-\frac{z}{\bar{\theta}-\underline{\theta}}: z \in L(x)\right\}
$$

if $x \in X$ and otherwise by

$$
J(x)= \begin{cases}J(\bar{x}) & \text { if } x>\bar{x} \\ J(\underline{x}) & \text { if } x<\underline{x}\end{cases}
$$

is a nonempty, compact and convex-valued upper hemicontinuous correspondence from $[\underline{x}-$ $1, \bar{x}+1]$ into itself, ${ }^{18}$ and hence by Kakutani's fixed point theorem has a fixed point (cf. Border [3, Corollary 15.3]). By construction, such a fixed point must occur at some $\hat{x} \in(\underline{x}, \bar{x})$ for which $0 \in L(\hat{x})$.

Proof of Proposition 2: The function $H$ given by (35) is continuously differentiable with

$$
H_{\hat{x}}(\hat{x}, \hat{\theta})=b\left(\hat{x}, \phi^{b}(\hat{x})\right)-s\left(\hat{x}, \phi^{s}(\hat{x})\right)+V_{x}(\hat{x})+u_{x}(\hat{x}, \hat{\theta}) .
$$

From Lemma 7, Assumption 2 implies that the value function $W$ defined in (34) satisfies

$$
W(\hat{x})=H(\hat{x}, \xi(\hat{x}))
$$

where $\xi: X \rightarrow \Theta$ is any selection from $\Theta_{\min }(x)$. Due to the single crossing property, $\xi$ is decreasing. Furthermore, $W$ is absolutely continuous with derivative

$$
W_{\hat{x}}(\hat{x})=H_{\hat{x}}(\hat{x}, \xi(\hat{x}))
$$

for almost all $\hat{x}$, implying that the condition (ignoring that inequality whose limit is undefined when considering $x^{*}=\underline{x}$ or $\left.x^{*}=\bar{x}\right)$

$$
\lim _{\hat{x} \uparrow x^{*}} H_{\hat{x}}(\hat{x}, \xi(\hat{x})) \geq 0 \geq \lim _{\hat{x} \downarrow x^{*}} H_{\hat{x}}(\hat{x}, \xi(\hat{x}))
$$

is necessary for $x^{*}$ to satisfy $x^{*} \in \arg \max _{\hat{x}} W(\hat{x})$. Assumption 1 implies that (41) holds if and only if there exists $\theta^{*} \in \Theta_{\min }\left(x^{*}\right)$ satisfying (36).

\footnotetext{
${ }^{18}$ Note that $z \in L(x)$ ensures $z /(\bar{\theta}-\underline{\theta}) \in[-1,1]$. By assumption, $\max L(\underline{x})<0$ and hence $J(\underline{x})>\underline{x}$, and $\min L(\bar{x})<0$ and hence $J(\bar{x})<\bar{x}$.
} 
Next, suppose that $H$ is pseudo-concave in $\hat{x}$ for all $\hat{\theta}$. Because $H_{\hat{x} \hat{\theta}}>0$ and $\xi$ is decreasing, (40) would then imply the pseudo-concavity of $W$ and thus the sufficiency of (41) for $x^{*} \in$ $\arg \max _{\hat{x}} W(\hat{x})$, completing the proof.

It thus remains to show that $H$ is pseudo-concave. Because $\phi^{b} \leq \phi^{s}$ and both of these type assignments are increasing, for any given $\hat{\theta}$ there exist $x_{1} \leq x_{2}$ such that

$$
\begin{aligned}
& \hat{\theta}>\phi^{s}(\hat{x}) \geq \phi^{b}(\hat{x}) \quad \text { if } \quad \hat{x}<x_{1} \\
& \phi^{b}(\hat{x}) \leq \hat{\theta} \leq \phi^{s}(\hat{x}) \quad \text { if } \quad \hat{x} \in\left(x_{1}, x_{2}\right) \\
& \phi^{s}(\hat{x}) \geq \phi^{b}(\hat{x})>\hat{\theta} \quad \text { if } \quad \hat{x}>x_{2} .
\end{aligned}
$$

Using (24) with $(x, \theta)=\left(\hat{x}, \phi^{s}(\hat{x})\right)$, we can rewrite (39) as follows, with (1) implying the inequality:

$$
H_{\hat{x}}(\hat{x}, \hat{\theta})=\left[b\left(\hat{x}, \phi^{b}(\hat{x})\right)-b\left(\hat{x}, \phi^{s}(\hat{x})\right)\right]+\left[u_{x}(\hat{x}, \hat{\theta})-u_{x}\left(\hat{x}, \phi^{s}(\hat{x})\right)\right]>0, \forall \hat{x}<x_{1} .
$$

An analogous argument with $(x, \theta)=\left(\hat{x}, \phi^{b}(\hat{x})\right)$ establishes

$$
H_{\hat{x}}(\hat{x}, \hat{\theta})=\left[s\left(\hat{x}, \phi^{b}(\hat{x})\right)-s\left(\hat{x}, \phi^{s}(\hat{x})\right)\right]+\left[u_{x}(\hat{x}, \hat{\theta})-u_{x}\left(\hat{x}, \phi^{b}(\hat{x})\right)\right]<0, \forall \hat{x}>x_{2}
$$

Using $(24)$ with $(x, \theta)=(\hat{x}, \hat{\theta})$, we can rewrite $(39)$ as:

$$
H_{\hat{x}}(\hat{x}, \hat{\theta})=\left[b\left(\hat{x}, \phi^{b}(\hat{x})\right)-b(\hat{x}, \hat{\theta})\right]-\left[s\left(\hat{x}, \phi^{s}(\hat{x})\right)-s(\hat{x}, \hat{\theta})\right] .
$$

From (42) and the definitions of $\phi^{b}$ and $\phi^{s}$, the function $H_{\hat{x}}$ is absolutely continuous in $\hat{x}$ with derivative

$$
H_{\hat{x} \hat{x}}(\hat{x}, \hat{\theta})=\left[b_{x}\left(\hat{x}, \phi^{b}(\hat{x})\right)-b_{x}(\hat{x}, \hat{\theta})\right]-\left[s_{x}\left(\hat{x}, \phi^{s}(\hat{x})\right)-s_{x}(\hat{x}, \hat{\theta})\right]
$$

for almost all $\hat{x}$. Because Assumption 2 implies $b_{x \theta}>0$ and $s_{x \theta}>0$ (cf. the proof of Lemma 6), (43) implies $H_{\hat{x} \hat{x}}(\hat{x}, \hat{\theta}) \leq 0$ for $\hat{x} \in\left(x_{1}, x_{2}\right)$, completing the argument establishing the pseudoconcavity of $H$. 


\section{References}

[1] D. Baron, R. Myerson, Regulating a Monopolist with Unknown Cost, Econometrica 50 (1982), 911-930.

[2] B. Biais, D. Martimort. J.-C. Rochet, Competing Mechanisms in a Common Value Environment, Econometrica 68 (2000), 799-838.

[3] K. C. Border, Fixed Point Theorems with Applications to Economics and Game Theory, Cambridge University Press, Cambridge, 1985.

[4] R. C. Feenstra, T. R. Lewis, Negotiated Trade Restrictions with Private Political Pressure, Quart. J. Econ. 106 (1991), 1287-1308.

[5] D. Fudenberg, J. Tirole, Game Theory, MIT Press, Cambridge, MA, 1991.

[6] L. R. Glosten, Insider Trading, Liquidity, and the Role of the Monopolist Specialist, J. Bus. 62 (1989), 211-235.

[7] L. R. Glosten, Is the Electronic Open Limit Order Book Inevitable? J. Finance 49 (1994), $1127-1161$.

[8] M. B. Goldman, H. E. Leland, D. S. Sibley, Optimal Nonuniform Pricing, Rev. Econ. Stud. 51 (1984), 305-320.

[9] R. Guesnerie, J.-J. Laffont, A Complete Solution to a Class of Principle-Agent Problems with an Application to the Control of a Self-Managed Firm, J. Public Econ. 25 (1984), $329-369$.

[10] B. Jullien, Participation Constraints in Adverse Selection Models, J. Econ. Theory 93 (2000), 1-48.

[11] J.-J. Laffont, J. Tirole, A Theory of Incentives in Procurement and Regulation, MIT Press, Cambridge, MA, 1993. 
[12] T. R. Lewis, D. E. M. Sappington, Inflexible Rules in Incentive Problems, Amer. Econ. Rev. 79 (1989), 69-84.

[13] T. R. Lewis, D. E. M. Sappington, Countervailing Incentives in Agency Problems, J. Econ. Theory 49 (1989), 294-313.

[14] G. Maggi, A. Rodriguez-Clare, On Countervailing Incentives, J. Econ. Theory 66 (1995), $238-263$.

[15] E. Maskin, J. Riley, Monopoly with Incomplete Information, RAND J. Econ. 15 (1984), $171-196$.

[16] P. Milgrom, C. Shannon, Monotone Comparative Statics, Econometrica 62 (1994), 157-180.

[17] P. Milgrom, I. Segal, Envelope Theorems for Arbitrary Choice Sets, Econometrica 70 (2002), 583-601.

[18] R. Myerson, Optimal Auction Design, Mathematics of Operations Research 6 (1981), 58-73.

[19] M. Mussa, S. Rosen, Monopoly and Product Quality, J. Econ. Theory 18 (1978), 301-317.

[20] J.-C. Rochet, The Taxation Principle and Multi-Time Hamilton-Jacobi Equations, Journal of Mathematical Economics 14 (1985), 113-128.

[21] J.-C. Rochet, A Necessary and Sufficient Condition for Rationalizability in a Quasi-Linear Context, J. Math. Econ. 16 (1987), 191-200.

[22] T. Rockafellar, Convex Analysis, Princeton University Press, Princeton, NJ, 1970.

[23] R. Strausz, Deterministic versus Stochastic Mechanisms in Principal-Agent Models, J. Econ. Theory, forthcoming.

[24] R. B. Wilson, Nonlinear Pricing, Oxford University Press, Oxford, 1993. 